\title{
A HARDWARE TESTBED FOR THE IMPLEMENTATION AND EVALUATION OF MIMO ALGORITHMS
}

\author{
PATRICK MURPHY, FEIFEI LOU and J. PATRICK FRANTZ \\ Rice University, 6100 Main Street, Houston, TX, 77005, USA \\ E-mail: \{murphpo, fflou, jpfrantz\}@rice.edu
}

\begin{abstract}
As the demand for higher performance wireless communications continues to grow, novel algorithms have been developed which provide increased performance and efficiency. One such class of algorithms involves the use of multiple antennas on either end of a wireless link. Many of these multiple input, multiple output (MIMO) algorithms offer impressive performance gains over their single antenna counterparts. The practicality of implementing such algorithms in a real system, however, is a topic in need of further exploration. We present in this paper a testbed designed specifically to test such algorithms. This testbed provides hardware for baseband processing, up and downconversion to RF and emulation of multiple wireless channels. It was designed to provide sufficient flexibility to implement a wide range of algorithms while preserving the ability to evaluate an algorithm's resource and power requirements. The testbed hardware and configuration options are presented, along with a basic demonstration of its functionality.
\end{abstract}

\section{Introduction}

The wireless communications industry has seen continuous growth in the demand for higher throughput communications. This increasing demand, however, has not been met with the availability of increased spectral resources. As a result, researchers have sought algorithms which provide higher performance without requiring additional allocation of spectrum.

As mentioned above, MIMO techniques encompass one class of algorithms which fit this requirement. There has been a great deal of effort spent developing and analytically evaluating MIMO algorithms. Far less effort, however, has been expended evaluating the challenges and practicality of implementing these algorithms in real hardware. This is due in part to the relative scarcity of hardware testbeds with sufficient resources to realize an endto-end, multiple antenna wireless link. We present a testbed designed explicitly to fill this void.

The remainder of this paper is organized as follows. Section 2 discusses the testbed design and the hardware chosen to construct it. Section 3 outlines the implementation of a simple MIMO scheme using the testbed's resources. Finally, section 4 offers some concluding remarks.

\section{Hardware}

The testbed consists of hardware which performs three major functions: baseband processing, conversion to and from radio frequencies and emulation of realistic wireless channels. The components we chose to fill each role are discussed below.

\subsection{Baseband Hardware}

One of the major challenges in designing a testbed capable of real-time wideband wireless communication is choosing baseband hardware. We first identified the major requirements the baseband hardware must meet. First, it must possess sufficient processing power to implement a wide variety of complex algorithms. Reliable wireless communication alone is a computationally intensive task; the added complexity of multiple transmit and receive antennas can increase the computational requirements significantly. The hardware must also allow reliable measurement of resource utilization and power consumption on a per-algorithm basis. This is an important requirement as the computational requirements of a particular algorithm play a large role in gauging its suitability for real-world use.

We found early on that of all the options for baseband processing, FPGAs provided the greatest flexibility in algorithm design and visibility of resource utilization. We acknowledge that FPGAs may not be ideal for deployment in large scale wireless systems. They are, however, very well suited for use in a rapid prototyping and research environment such as this testbed.

We decided on FPGA-based development systems designed by Nallatech of Glasgow, Scotland as our baseband processing platform. As shown in Figure 1 , each board houses a multi-million gate Xilinx 
Virtex-II FPGA and two channels each of analog-todigital and digital-to-analog conversion. The board's ADCs and DACs are fast enough to connect directly to the intermediate frequency interfaces on the testbed's RF hardware. These connections allow each board to potentially act as a dual-antenna transceiver. Each board also contains a smaller FPGA dedicated to providing clocks to the larger FPGA and the external analog interfaces. This feature provides a great deal of flexibility in connecting to hardware from different designers with various interface requirements. Finally, the Nallatech board is also a PCI card, providing a high throughput connection over which a host $\mathrm{PC}$ can provide and receive data and monitor system performance.

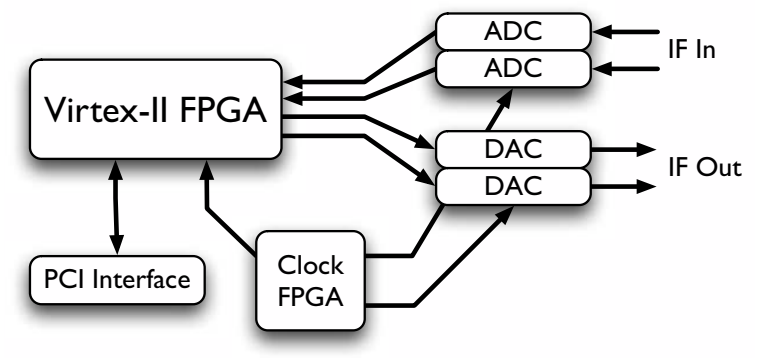

Figure 1. FPGA-based processing board

\subsection{RF Hardware}

This testbed is designed for wideband wireless communication in the $2.4 \mathrm{GHz}$ ISM band. The RF hardware must not be tied to any particular standard and must have intermediate frequencies within the sampling capability of the FPGA baseband platform. We also desired control over the synchronization of the frequency translation stages of the radios. Such control allows variation in the carrier frequency offsets between antennas and will prove useful in characterizing the performance of multi-antenna carrier recovery schemes. It also allows offsets between the carriers of multiple transmit or receive antennas to be eliminated entirely, a useful option when testing algorithms lacking carrier recovery systems.

We decided on $2.4 \mathrm{GHz} \mathrm{RF}$ hardware from National Instruments. NI has only recently ventured into the RF hardware market, but we found that their hardware fit the needs of our testbed very well. These devices operate with an intermediate frequency of just $15 \mathrm{MHz}$, easily within range of the
FPGA baseband's sampling capabilities. They also support signal bandwidths up to $20 \mathrm{MHz}$, accommodating a large variety of wideband schemes.

In order to maximize flexibility in choosing multiple antenna configurations, the testbed also uses a number of RF multiplexers. These devices form the interconnect between the $\mathrm{RF}$ transceivers and the channel emulators, described in section 2.3. The multiplexers allow most configurations to be implemented programmatically without any changes to the equipment's physical connections. This both minimizes the risk of damage to the equipment and allows for a great deal of automation in switching between interconnection configurations.

\subsection{Wireless Channel Emulation}

Another significant challenge in designing this wireless testbed was identifying some means by which we could provide a realistic wireless channel in a laboratory setting. We required the ability to simulate a wide variety of channel conditions, including fading, multi-path effects and delay spread. We also needed to assure repeatability of experimental results, a virtually impossible requirement when using real wireless channels.

To fill this need, we chose RF channel emulators from Spirent Communications. Each device is capable of emulating two wireless channels, each with six independently configured paths. Figure 2 illustrates the capabilities of each emulated wireless channel.

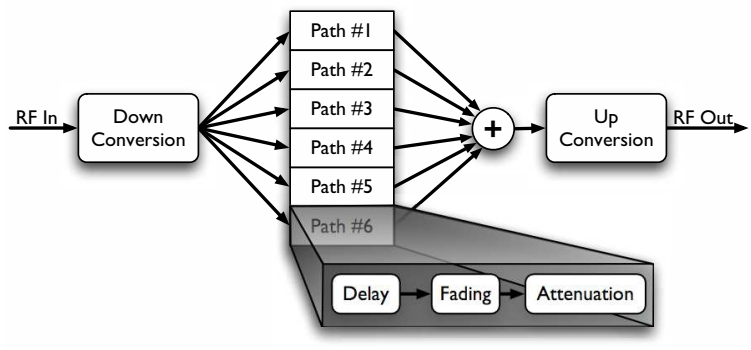

Figure 2. Emulated wireless channel

The emulator can apply one of many fading models, including Rayleigh, Rician and Nakagami, plus programmed delay and attenuation to each path. This flexibility allows the emulation of a large number of realistic line-of-sight and multi-path environments. The emulator can also introduce arbitrary frequency shifts to each path, providing a reliable 


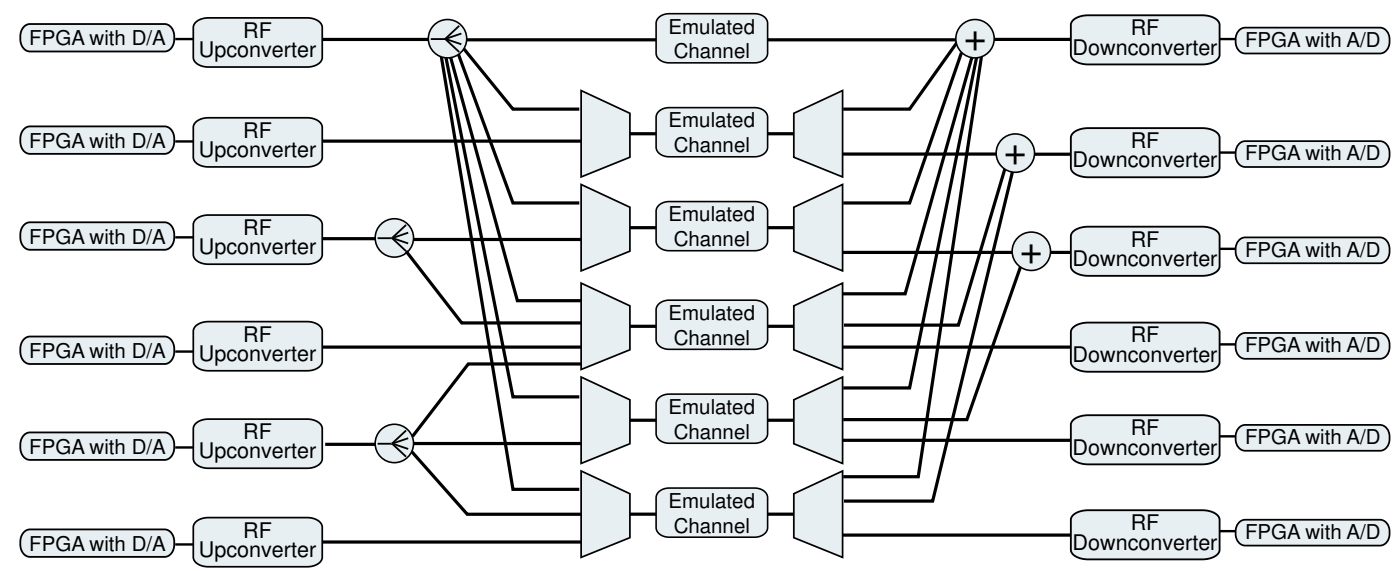

Figure 3. Testbed connectivity for half-duplex testing

means by which to simulate the Doppler effects of various mobile scenarios.

Our testbed currently houses three such emulators, providing a total of six independent emulated channels. This fairly large number of channels provides significant flexibility in designing test configurations. In terms of transmit-to-receive antennas, the testbed can implement half-duplex systems of 6-to-1, 3-to-2, 2-to-3, 1-to-6 or any smaller configuration requiring six or fewer channels. Figure 3 illustrates the full connectivity for multiple-antenna half-duplex testing.

\section{Example Application}

The final stage of funding for this testbed has only recently been approved. As a result, the first projects which utilize its resources are still under development and will be the focus of future publications. However, in order to demonstrate the testbed's functionality, we offer the implementation of the simple algorithm utilizing multiple transmit antennas first described by Alamouti [1]. This scheme is well suited for such a demonstration as it uses every stage of the testbed but is fairly straightforward to implement.

Alamouti's technique is a transmit diversity scheme utilizing, at a minimum, two transmit antennas and one receive antenna. It is an example of a space-time block code, a multiple antenna technique which requires only linear processing in the encoding and decoding states to achieve its diversity gain [2].

Our implementation effort began with the selection of Xilinx's System Generator for DSP [3]. Sys- tem Generator is an extension for The Mathworks' Simulink environment which allows models to be rapidly constructed, simulated and synthesized for use in real FPGA hardware.

Our model currently consists of a transmitter and receiver in the same FPGA. The transmitter is fairly simple, consisting of a QPSK modulator, Alamouti encoder, pulse shaping filter and digital upconverter for translation to IF.

The receiver is substantially more complicated. It includes subsystems for carrier recovery, channel estimation, decoding and demodulation. The carrier recovery block assumes the transmit antennas share a reference clock and have no offset. It then corrects for any frequency offset between the transmit and receive radios. The channel estimation system correlates the received data against a known training sequence to estimate each channel's phase offset and attenuation. The channel estimates are used until the training sequence is transmitted again. This approach assumes both channels have slowly varying characteristics, a limitation that will be eliminated with more sophisticated algorithms in future revisions of the design.

Top level views of the System Generator design for the Alamouti transmitter and receiver are shown in Figures 4 and 5. The design has been synthesized and implemented in a two million gate Xilinx Virtex-II FPGA (XC2V2000). The current revisions of the transmitter and receiver together utilize approximately $85 \%$ of the logic resources in the FPGA. This utilization should be viewed as an upper bound as there exist a variety of possible optimiza- 


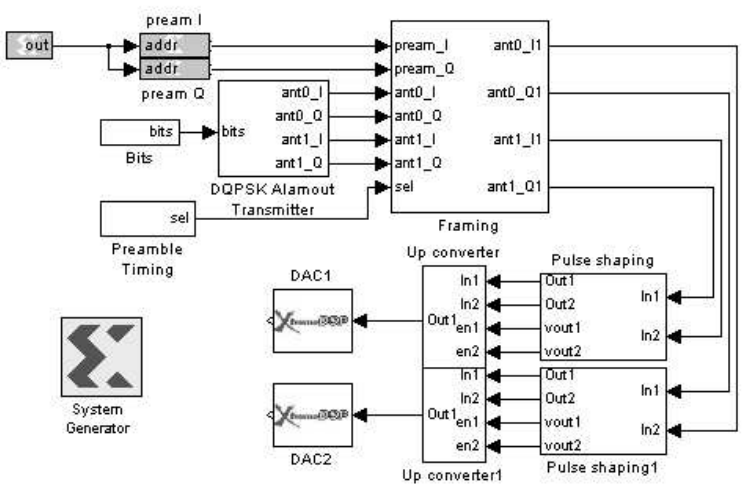

Figure 4. System Generator model of Alamouti transmitter

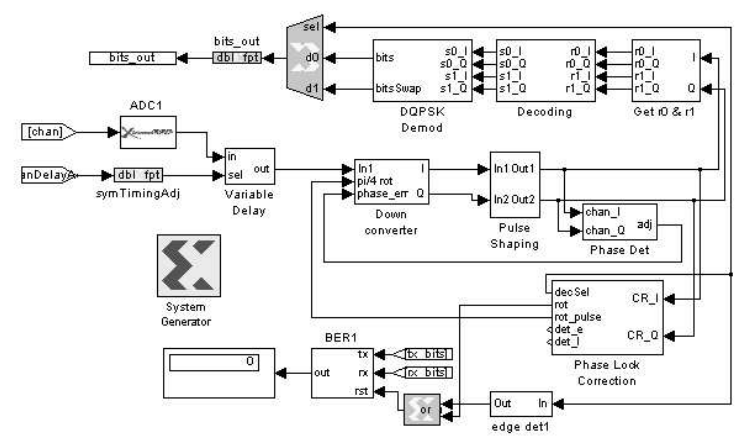

Figure 5. System Generator model of Alamouti receiver

tions not yet applied to the design. The system has been tested as an end-to-end link using the baseband, $\mathrm{RF}$ and channel emulation hardware described above with realized throughputs in excess of $1 \mathrm{Mb} / \mathrm{sec}$.

\section{Conclusion}

There is little doubt that many future wireless communications systems will employ multiple antenna techniques to provide high performance while maximizing spectral efficiency. This expected prevalence of MIMO systems highlights the need for platforms designed to evaluate such algorithms under realistic conditions. The practical value of an algorithm is increased significantly when it is tested in real hardware and evaluated in terms of computational requirements and power consumption. This paper has described in detail the hardware platform we have constructed to perform this analysis.

\section{Acknowledgments}

The equipment for this testbed was purchased in part with NSF grants CISE-ANI-0224458 and CISE-MRI0321266. Authors Murphy and Lou are each partly supported by Texas Instruments Fellowships. Special thanks to Xilinx, Nallatech, Spirent Communications and National Instruments for their generous equipment discounts and donations.

\section{References}

1. S. M. Alamouti, "A simple transmit diversity technique for wireless communications," IEEE Journal on Select Areas in Comm., vol. 16, pp. 1451-1458, Oct. 1998.

2. V. Tarokh, H. Jafarkhani, A.R. Calderbank, "Space-time block codes from orthogonal designs," IEEE Transactions on Information Theory, vol. 45, pp. 1456-1467 July 1999.

3. http://www.xilinx.com/system_generator/ 